\title{
Effects of pH on the Speciation of Radionuclides in Groundwater of Beishan Area, Gansu Province, China
}

\author{
Zhang Zhanshi* Zhou Wenbin* Fan Xianhua **
}

\begin{abstract}
Speciation of radioactive nuclides is one of the most important factors in the study of migration and precipitation of the nuclides related to deep geological disposal of high level radwastes (HLW). Theoretical speciation analysis with thermodynamic equilibrium codes is an effective method. The authors have employed the EQ3NR, a computer program for geochemical aqueous speciation-solubility calculation, to evaluate the speciation of the key nuclides in the groundwater of Beishan Area, a potential area for hosting the HLW Repository of China. The calculated results showed that $\mathrm{pH}$ value as well as chemical composition of the water had a great impact on the speciation of key nuclides, such as $\mathrm{Np}, \mathrm{Pu}, \mathrm{Am}, \mathrm{U}$ and Th, which would be released from HLW to the groundwater of Beishan Area. In addition, it was found that the speciation of nuclides varied from one to another. However, under alkaline conditions, the speciations were relatively simpler and hydroxyl complexes and carbonate complexes were dominant. Key words: Geochemical modeling, Speciation, High level waste disposal, EQ3NR
\end{abstract}

\section{INTRODUCTION}

The national project on deep geological disposal (DGD) of HLW in China has been carried out for 15 years. After the national, regional and area screening, Beishan Area of Gansu Province in West China has been selected as a potential area for hosting the repository of HLW in China. Now, preliminary site characterization, including geological, hydrogeological, and geotechnical investigations, is on its way[1].

The speciation of radioactive nuclides in groundwater is one of the most important factors in the study of nuclide migration, which is a key factor in performance assessment of a repository. On the basis of hydrogeological and hydrogeochemical survey in Beishan area, the effects of $\mathrm{pH}$ on the speciation and solubility of several key radioactive nuclides are simulated by using EQ3NR.

\section{CALCULATION CONDITIONS}

Speciation and solubility calculation of nuclides were performed with the geochemical codes EQ3NR (Version 7.2a), a computer program for geochemical aqueous speciation-solubility calculation, developed by Wolery of Lawrence Livermore National Laboratory (LLNL). It is part of the EQ3/6 software package for geochemical modeling [2-3]. The attached thermodynamic data file is employed in this study. The numerical method employed is based on a hybrid Newton-Raphson technique, and the B-dot equation has been used for calculating activity coefficients. The nuclide concentrations adopted are $10^{-12} \mathrm{~mol} / \mathrm{L}$ and the temperature adopted is $25^{\circ} \mathrm{C}$. All the species of related nuclides in the attached data file are listed in Table 1. The emphasis is on the effects of $\mathrm{pH}$ on the speciation and solubility of nuclides in this study.

This article was presented in "Japan-China Workshop on Nuclear Waste Management and Reprocessing".

Effects of $\mathrm{pH}$ on the Speciation of Radionuclides in Groundwater of Beishan Area, Gansu Province, China by Zhang Zhanshi (zszhang@ ecgi.jx.cn), Zhou Wenbin, Fan Xianhua

* East China Institute of Technology, Fuzhou, Jiangxi, 344000, China

* * Radiochemistry Department, China Institute of Atomic Energy, Beijing,

102413, China

\section{CHEMICAL CHARACTERISTICS OF GROUND- WATER IN BEISHAN AREA}

Beishan, a potential area for the HLW repository in Gansu Province of West China, is located in a typical arid climate region. As a part of the HLW DGD project of China, a preliminary characterization of hydrogeology and chemistry of the groundwater for the area has been carried out. The $\mathrm{pH}$ values for the shallow groundwater in this area are usually in the range of 7.1 to 8.8 , temperature from 8 to $14^{\circ} \mathrm{C}$, and the total dissolved solid (TDS) from 0.3 to $12 \mathrm{~g} / \mathrm{L}$. The majority of shallow groundwater is of $\mathrm{Cl}-\mathrm{SO}_{4}-\mathrm{Na}$ and $\mathrm{SO}_{4}$-Cl-Na type , followed by $\mathrm{Cl}-\mathrm{SO}_{4}-\mathrm{Na}-\mathrm{Ca}$ type[4]. The water from Wuyi well, a 50-meter-deep well in Beishan area, is considered as the representative of the deep groundwater for the area. The chemical composition of the water, shown in Table 2, has been used in the modeling [4].

\section{RESULTS AND DISCUSSIONS}

\subsection{Speciation of Radionuclides in Beishan Groundwater}

Table 3 shows the speciations of the critical radionuclides in the groundwater of Beishan Wuyi well. The speciations of $\mathrm{Am}, \mathrm{Np}$ and $\mathrm{U}$, which are most hazardous radionuclides in HLW, are shown in Figs. 1 to 3.

Figure 1 indicates that in the water of Beishan Wuyi well, the dominant species of $\mathrm{Am}$ is $\mathrm{AmCO}_{3}{ }^{+}(84.42 \%)$ and the other species of $\mathrm{Am}$ are $10.15 \%$ of $\mathrm{Am}\left(\mathrm{CO}_{3}\right)_{2}{ }^{-}, 2.58 \%$ of $\mathrm{AmOH}^{2+}$, $1.69 \%$ of $\mathrm{AmSO}_{4}^{+}$and $0.941 \%$ of $\mathrm{Am}^{3+}$.

From Fig.2, the dominant species of $\mathrm{Pu}$ is $\mathrm{PuO}_{2}\left(\mathrm{CO}_{3}\right)_{2}{ }^{2-}$ $(71.88 \%)$, and the others are $\mathrm{PuO}_{2}^{+}(23.93 \%), \mathrm{PuO}_{2} \mathrm{OH}^{+}$ (2.13\%), $\mathrm{PuO}_{2} \mathrm{~F}_{3}{ }^{-}(0.802 \%)$ and $\mathrm{PuO}_{2} \mathrm{~F}_{2}$ (aq) (0.667\%).

Figure 3 indicates that the dominant species of $\mathrm{Np}$ in the groundwater of Beishan Wuyi well is $\mathrm{NpO}_{2}^{+}(93.76 \%)$. The minor species are $\mathrm{NpO}_{2} \mathrm{CO}_{3}{ }^{-}(4.09 \%), \mathrm{NpO}_{2} \mathrm{OH}(\mathrm{aq})(1.84 \%)$. 
Table 1 The species of related nuclides in attached data file in EQ3NR ${ }^{[2,3]}$

\begin{tabular}{|c|c|c|c|}
\hline $\begin{array}{l}\mathrm{Am}^{3+} \\
\mathrm{Am}\left(\mathrm{H}_{2} \mathrm{PO}_{4}\right)_{2}^{+} \\
\mathrm{Am}\left(\mathrm{H}_{2} \mathrm{PO}_{4}\right)_{3}(\mathrm{aq}) \\
\mathrm{Am}\left(\mathrm{H}_{2} \mathrm{PO}_{4}\right)_{4}^{-} \\
\mathrm{AmH}_{2} \mathrm{PO}_{4}^{2+}\end{array}$ & $\begin{array}{l}\mathrm{AmF}^{2+} \\
\mathrm{AmF}_{2}^{+} \\
\mathrm{AmF}_{3}(\mathrm{aq}) \\
\mathrm{AmCl}^{2+} \\
\mathrm{AmCl}_{2}^{+} \\
\end{array}$ & $\begin{array}{l}\mathrm{AmO}_{2} \mathrm{HCO}_{3}(\mathrm{aq}) \\
\mathrm{Am}\left(\mathrm{CO}_{3}\right)_{3}{ }^{3-} \\
\mathrm{AmCO}_{3}{ }^{+} \\
\mathrm{Am}\left(\mathrm{NO}_{3}\right)_{2}{ }^{+} \\
\mathrm{AmNO}_{3}{ }^{2+} \\
\end{array}$ & $\begin{array}{l}\mathrm{AmOH}^{2+} \\
\mathrm{Am}(\mathrm{OH})_{2}^{+} \\
\mathrm{Am}(\mathrm{OH})_{3}(\mathrm{aq}) \\
\mathrm{AmSO}_{4}{ }^{+} \\
\mathrm{Am}\left(\mathrm{SO}_{4}\right)_{2}^{-} \\
\end{array}$ \\
\hline $\mathrm{NpO}_{2}{ }^{+}$ & $\mathrm{NpF}^{3+}$ & 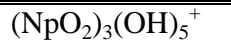 & $\mathrm{NpO}_{2} \mathrm{CO}_{3}^{-}$ \\
\hline $\mathrm{Np}\left(\mathrm{HPO}_{4}\right)_{4}{ }^{4-}$ & $\mathrm{NpF}_{2}^{2+}$ & $\left(\mathrm{NpO}_{2}\right)_{2}(\mathrm{OH})_{2}{ }^{2+}$ & $\mathrm{NpO}_{2}\left(\mathrm{CO}_{3}\right)_{2}{ }^{2-}$ \\
\hline $\mathrm{Np}\left(\mathrm{HPO}_{4}\right)_{5}^{6-}$ & $\mathrm{NpO}_{2} \mathrm{~F}(\mathrm{aq})$ & $\mathrm{Np}(\mathrm{OH})_{2}{ }^{2+}$ & $\mathrm{NpO}_{2}\left(\mathrm{CO}_{3}\right)_{3}{ }^{4-}$ \\
\hline $\mathrm{Np}\left(\mathrm{H}_{2} \mathrm{PO}_{4}\right)_{2}^{+}$ & $\mathrm{NpCl}^{3+}$ & $\mathrm{Np}(\mathrm{OH})_{3}{ }^{+}$ & $\mathrm{NpO}_{2}\left(\mathrm{CO}_{3}\right)_{2}^{3-}$ \\
\hline $\mathrm{Np}\left(\mathrm{H}_{2} \mathrm{PO}_{4}\right)_{3}(\mathrm{aq})$ & $\mathrm{NpCl}_{2}{ }^{2+}$ & $\mathrm{Np}(\mathrm{OH})_{4}(\mathrm{aq})$ & $\mathrm{Np}\left(\mathrm{CO}_{3}\right)_{5}^{6-}$ \\
\hline $\mathrm{Np}\left(\mathrm{HPO}_{4}\right)_{2}(\mathrm{aq})$ & $\mathrm{NpO}_{2} \mathrm{Cl}^{+}$ & $\mathrm{Np}(\mathrm{OH})_{5}^{-}$ & $\mathrm{NpSO}_{4}{ }^{2+}$ \\
\hline $\mathrm{NpO}_{2} \mathrm{H}_{2} \mathrm{PO}_{4}(\mathrm{aq})$ & $\mathrm{NpH}_{2} \mathrm{PO}_{4}{ }^{2+}$ & $\mathrm{NpO}_{2} \mathrm{OH}(\mathrm{aq})$ & $\mathrm{NpO}_{2} \mathrm{SO}_{4}^{-}$ \\
\hline $\mathrm{NpO}_{2} \mathrm{H}_{2} \mathrm{PO}_{4}^{+}$ & $\mathrm{NpO}_{2} \mathrm{HPO}_{4}(\mathrm{aq})$ & $\mathrm{NpO}_{2} \mathrm{OH}^{+}$ & $\mathrm{Np}\left(\mathrm{SO}_{4}\right)_{2}(\mathrm{aq})$ \\
\hline $\mathrm{NpO}_{2}{ }^{2+}$ & $\mathrm{NpO}_{2} \mathrm{HPO}_{4}^{-}$ & $\mathrm{NpOH}^{3+}$ & $\mathrm{NpO}_{2} \mathrm{SO}_{4}(\mathrm{aq})$ \\
\hline $\mathrm{PuO}_{2}^{+}$ & $\mathrm{PuO}_{2}{ }^{2+}$ & & \\
\hline $\mathrm{PuO}_{2} \mathrm{~F}^{+}$ & $\mathrm{PuO}_{2}\left(\mathrm{CO}_{3}\right)_{2}{ }^{2-}$ & $\mathrm{PuO}_{2} \mathrm{SO}_{4}(\mathrm{aq})$ & $\mathrm{PuO}_{2} \mathrm{OH}(\mathrm{aq})$ \\
\hline $\mathrm{PuF}_{2}^{2+}$ & $\mathrm{PuH}_{2} \mathrm{PO}_{4}{ }^{2+}$ & $\mathrm{PuSO}_{4}^{+}$ & $\mathrm{PuO}_{2} \mathrm{OH}^{+}$ \\
\hline $\mathrm{PuF}_{3}^{+}$ & $\mathrm{PuHPO}_{4}{ }^{2+}$ & $\mathrm{PuSO}_{4}{ }^{2+}$ & $\mathrm{Pu}(\mathrm{OH})_{5}^{-}$ \\
\hline $\mathrm{PuF}_{4}(\mathrm{aq})$ & $\mathrm{PuO}_{2} \mathrm{H}_{2} \mathrm{PO}_{4}^{+}$ & $\mathrm{Pu}\left(\mathrm{SO}_{4}\right)_{2}(\mathrm{aq})$ & $\mathrm{Pu}(\mathrm{OH})_{2}{ }^{2+}$ \\
\hline $\mathrm{PuF}^{3+}$ & $\mathrm{Pu}\left(\mathrm{HPO}_{4}\right)_{3}{ }^{2-}$ & $\mathrm{Pu}\left(\mathrm{SO}_{4}\right)_{2}^{-}$ & $\mathrm{Pu}(\mathrm{OH})_{3}{ }^{+}$ \\
\hline $\mathrm{PuO}_{2} \mathrm{~F}_{2}(\mathrm{aq})$ & $\mathrm{Pu}\left(\mathrm{HPO}_{4}\right)_{4}{ }^{4-}$ & $\mathrm{PuCl}^{3+}$ & $\mathrm{PuOH}^{3+}$ \\
\hline $\mathrm{PuO}_{2} \mathrm{~F}_{3}^{-}$ & $\mathrm{PuO}_{2} \mathrm{~F}_{4}^{2-}$ & $\mathrm{Pu}(\mathrm{OH})_{4}(\mathrm{aq})$ & $\mathrm{PuOH}^{2+}$ \\
\hline$\overline{\mathrm{Th}^{4+}}$ & $\overline{\mathrm{T}} \mathrm{Th}\left(\mathrm{H}_{2} \mathrm{PO}_{4}\right)_{2}{ }^{2+}$ & $\mathrm{CThSO}_{4}{ }^{2+}$ & $\mathrm{Th}(\mathrm{OH})_{2}{ }^{2+}$ \\
\hline $\mathrm{ThF}^{3+}$ & $\mathrm{Th}\left(\mathrm{HPO}_{4}\right)_{2}(\mathrm{aq})$ & $\mathrm{Th}\left(\mathrm{SO}_{4}\right)_{4}^{4-}$ & $\mathrm{Th}(\mathrm{OH})_{4}(\mathrm{aq})$ \\
\hline $\mathrm{ThF}_{2}^{2+}$ & $\mathrm{Th}\left(\mathrm{HPO}_{4}\right)_{3}{ }^{2-}$ & $\mathrm{Th}\left(\mathrm{SO}_{4}\right)_{2}(\mathrm{aq})$ & $\mathrm{Th}_{2}(\mathrm{OH})_{2}{ }^{6+}$ \\
\hline $\mathrm{ThF}_{3}^{+}$ & $\mathrm{ThH}_{2} \mathrm{PO}_{4}{ }^{3+}$ & $\mathrm{Th}\left(\mathrm{SO}_{4}\right)_{3}{ }^{2-}$ & $\mathrm{Th}_{4}(\mathrm{OH})_{8}{ }^{8+}$ \\
\hline $\mathrm{ThF}_{4}(\mathrm{aq})$ & $\mathrm{ThH}_{3} \mathrm{PO}_{4}{ }^{4+}$ & $\mathrm{ThCl}_{3}^{+}$ & $\mathrm{Th}_{6}(\mathrm{OH})_{15}{ }^{9+}$ \\
\hline $\mathrm{ThCl}^{3+}$ & $\mathrm{ThCl}_{4}(\mathrm{aq})$ & $\mathrm{ThCl}_{2}^{2+}$ & $\mathrm{ThOH}^{3+}$ \\
\hline $\mathrm{UO}_{2}{ }^{2+}$ & $\mathrm{U}\left(\mathrm{CO}_{3}\right)_{4}{ }^{4-}$ & $\mathrm{UO}_{2} \mathrm{OH}^{+}$ & 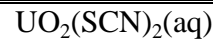 \\
\hline $\mathrm{UF}^{3+}$ & $\mathrm{U}\left(\mathrm{CO}_{3}\right)_{5}{ }^{6-}$ & $\mathrm{UOH}^{3+}$ & $\mathrm{UO}_{2}(\mathrm{SCN})_{3}{ }^{-}$ \\
\hline $\mathrm{UF}_{2}^{2+}$ & $\mathrm{UO}_{2}\left(\mathrm{CO}_{3}\right)_{2}{ }^{2-}$ & $\mathrm{U}(\mathrm{OH})_{4}(\mathrm{aq})$ & $\mathrm{UO}_{2} \mathrm{SCN}^{+}$ \\
\hline $\mathrm{UF}_{3+}$ & $\mathrm{UO}_{2}\left(\mathrm{CO}_{3}\right)_{3}^{-}$ & $\mathrm{UO}_{2}(\mathrm{OH})_{2}(\mathrm{aq})$ & $\mathrm{U}(\mathrm{SCN})_{2}{ }^{2+}$ \\
\hline $\mathrm{UF}_{4}(\mathrm{aq})$ & $\mathrm{UO}_{2}\left(\mathrm{CO}_{3}\right)_{3}^{4-}$ & $\mathrm{UO}_{2}(\mathrm{OH})_{3}^{-}$ & $\mathrm{USCN}^{3+}$ \\
\hline $\mathrm{UF}_{5}^{-}$ & $\mathrm{UO}_{2} \mathrm{CO}_{3}(\mathrm{aq})$ & $\mathrm{UO}_{2}(\mathrm{OH})_{4}{ }^{2-}$ & $\mathrm{UO}_{2}\left(\mathrm{~N}_{3}\right)_{2}(\mathrm{aq})$ \\
\hline $\mathrm{UF}_{6}{ }^{2-}$ & $\mathrm{UO}_{2}\left(\mathrm{H}_{2} \mathrm{PO}_{4}\right)\left(\mathrm{H}_{3} \mathrm{PO}_{4}\right)^{+}$ & $\mathrm{UO}_{2}\left(\mathrm{SO}_{3}\right)_{2}{ }^{2-}$ & $\mathrm{UO}_{2}\left(\mathrm{~N}_{3}\right)_{3}^{-}$ \\
\hline $\mathrm{UO}_{2} \mathrm{~F}^{+}$ & $\mathrm{UO}_{2}\left(\mathrm{H}_{2} \mathrm{PO}_{4}\right)\left(\mathrm{H}_{3} \mathrm{PO}_{4}\right)^{+}$ & $\mathrm{UO}_{2}\left(\mathrm{SO}_{4}\right)_{2}{ }^{2-}$ & $\mathrm{UO}_{2}\left(\mathrm{~N}_{3}\right)_{4}{ }^{2-}$ \\
\hline $\mathrm{UO}_{2} \mathrm{~F}_{2}(\mathrm{aq})$ & $\mathrm{UO}_{2}\left(\mathrm{H}_{2} \mathrm{PO}_{4}\right)_{2}(\mathrm{aq})$ & $\mathrm{UO}_{2} \mathrm{IO}_{3}^{+}$ & $\mathrm{UNO}_{3}{ }^{3+}$ \\
\hline $\mathrm{UO}_{2} \mathrm{~F}_{3}^{-}$ & $\mathrm{UO}_{2} \mathrm{H}_{2} \mathrm{PO}_{4}^{+}$ & $\mathrm{UO}_{2}\left(\mathrm{IO}_{3}\right)_{2}(\mathrm{aq})$ & $\mathrm{U}\left(\mathrm{NO}_{3}\right)_{2}{ }^{2+}$ \\
\hline $\mathrm{UO}_{2} \mathrm{~F}_{4}^{2-}$ & $\mathrm{UO}_{2} \mathrm{H}_{3} \mathrm{PO}_{4}^{2+}$ & $\mathrm{UI}^{3+}$ & $\mathrm{UO}_{2} \mathrm{~N}_{3}^{+}$ \\
\hline $\mathrm{UO}_{2} \mathrm{Cl}^{+}$ & $\mathrm{UO}_{2} \mathrm{HPO}_{4}(\mathrm{aq})$ & $\mathrm{UO}_{2} \mathrm{~S}_{2} \mathrm{O}_{3}(\mathrm{aq})$ & $\mathrm{UO}_{2} \mathrm{NO}_{3}^{+}$ \\
\hline $\mathrm{UO}_{2} \mathrm{Cl}_{2}(\mathrm{aq})$ & $\mathrm{UO}_{2} \mathrm{PO}_{4}^{-}$ & $\mathrm{UO}_{2} \mathrm{SO}_{3}(\mathrm{aq})$ & $\mathrm{UBr}^{3+}$ \\
\hline $\mathrm{UO}_{2} \mathrm{ClO}_{3}^{+}$ & & $\mathrm{UO}_{2} \mathrm{SO}_{4}(\mathrm{aq})$ & $\mathrm{UO}_{2} \mathrm{Br}^{+}$ \\
\hline $\mathrm{UCl}^{3+}$ & & $\mathrm{U}\left(\mathrm{SO}_{4}\right)_{2}(\mathrm{aq})$ & $\mathrm{UO}_{2} \mathrm{BrO}_{3}^{+}$ \\
\hline
\end{tabular}

Table 2 Chemical composition of water from Wuyi well in Beishan Area[4]

\begin{tabular}{cc||cc||cc||cc}
\hline Element & Concentration & Element & Concentration & Element & Concentration & Element & Concentration \\
\hline $\mathrm{Na}^{+}$ & 47.83 & $\mathrm{NH}_{4}^{+}$ & 0.12 & $\mathrm{HCO}_{3}{ }^{-}$ & 103.7 & $\mathrm{SiO}_{2}(\mathrm{aq})$ & 12.91 \\
$\mathrm{Ca}^{2+}$ & 73.88 & $\mathrm{Al}^{3+}$ & 0.06 & $\mathrm{Cl}^{-}$ & 61.35 & $\mathrm{NO}_{3}^{-}$ & 10.42 \\
$\mathrm{~K}^{+}$ & 8.88 & $\mathrm{Mn}^{2+}$ & 0.022 & $\mathrm{~F}^{-}$ & 0.26 & Eh $(\mathrm{V})$ & 0.345 \\
$\mathrm{Mg}^{2+}$ & 8.98 & $\mathrm{Li}^{+}$ & 0.0112 & $\mathrm{Br}^{-}$ & 0.0001 & $\mathrm{pH}$ & 7.24 \\
$\mathrm{Cu}$ & 0.0001 & $\mathrm{Sr}^{2+}$ & 0.715 & $\mathrm{SO}_{4}{ }^{2-}$ & 161.8 & & \\
\hline
\end{tabular}

Note: Measured at the $50 \mathrm{~m}$ deep in-situ condition, $\mathrm{mg} / \mathrm{L}$ 


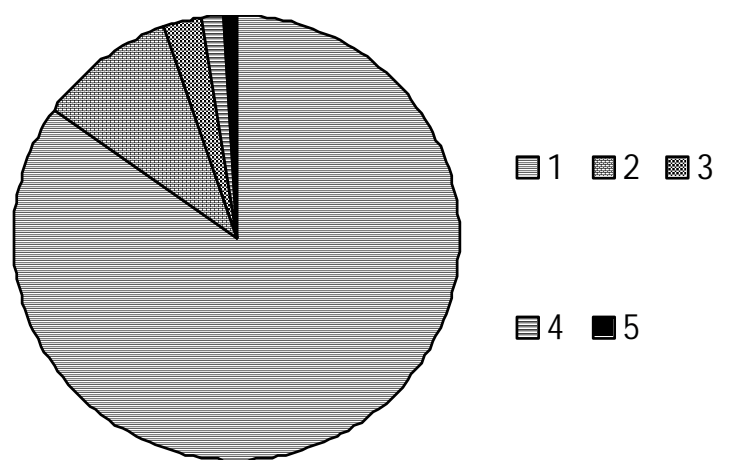

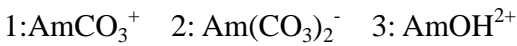

$$
\begin{aligned}
& \text { 4: } \mathrm{AmSO}_{4}^{+} \text {5: } \mathrm{Am}^{3+}
\end{aligned}
$$

Fig.1 Speciation of Am in the groundwater

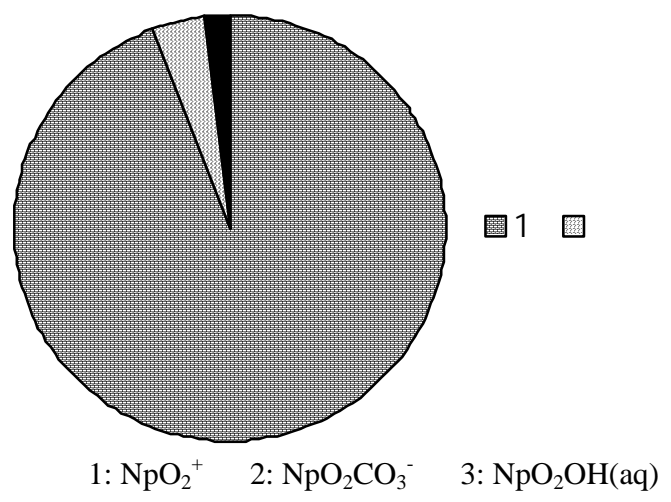

Fig.3 Speciation of $\mathrm{Np}$ in the groundwater

\subsection{Solubility of nuclides under in-situ condition}

Saturation indices(SI's) are important because they are measures of the thermodynamic driving forces behind the progress of reaction. These quantities form an important bridge between calculations that are purely thermodynamic and those that pertain to dynamic evolution. The saturation index (SI) is defined as : $\mathrm{SI}=\log \mathrm{Q} / \mathrm{K}$, where $\mathrm{Q}$ is the activity product and $\mathrm{K}$ the equilibrium constant for a dissolution reaction [2,3]. Following the above definition, saturation indices are zero for the case of exact saturation, negative for undersaturation, and positive for supersaturation. So the SI of a mineral could be used to discuss relative solubility of the nuclides in solution even though the concentration of actinides is assumed to be $10^{-12} \mathrm{~mol} / \mathrm{L}$. The higher the SI of a mineral is, the lower the solubility is.

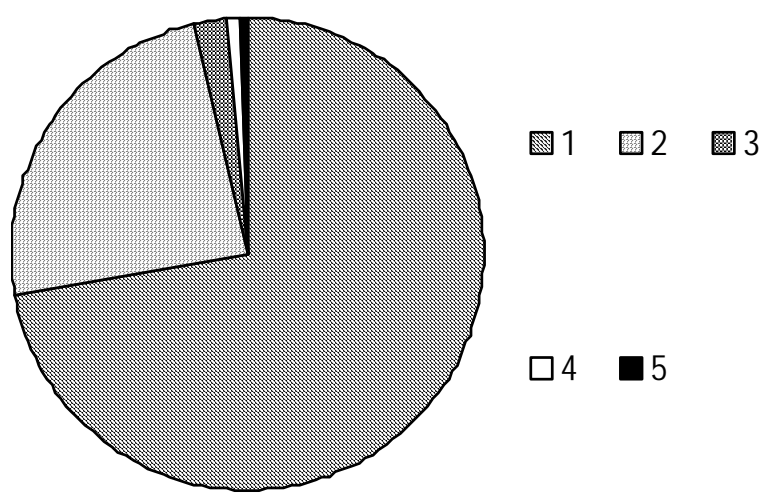

1: $\mathrm{PuO}_{2}\left(\mathrm{CO}_{3}\right)_{2}{ }^{2-} \quad$ 2: $\mathrm{PuO}_{2}{ }^{+} \quad$ 3: $\mathrm{PuO}_{2} \mathrm{OH}^{+}$
4: $\mathrm{Pu} \mathrm{O}_{2} \mathrm{~F}_{3}^{-} \quad$ 5: $\mathrm{PuO}_{2} \mathrm{~F}_{2}(\mathrm{aq})$

Fig.2 Speciation of $\mathrm{Pu}$ in the groundwater

Table 4 shows all the SI values of minerals containing the nuclides in decreasing order. The SI values of $\mathrm{PuO}_{2}$, Thorianite and $\mathrm{NpO}_{2}$ are higher than zero in Beishan Wuyi well. Those minerals would be precipitated and should not dissolve over $10^{-12} \mathrm{~mol} / \mathrm{L}$ at that condition. Other minerals such as $\mathrm{AmOHCO}_{3}$ are hardly formed and the solubility will be higher than $10^{-12} \mathrm{~mol} / \mathrm{L}$.

\subsection{Effects of $\mathrm{pH}$ on the speciation of nuclide}

The speciation of nuclides is greatly affected by the $\mathrm{pH}$ value. In this study a great emphasis is put on the effects of $\mathrm{pH}$ on the speciation and solubility of nuclides.

\subsubsection{Speciation of $\mathrm{Np}$ as a function of $\mathrm{pH}$}

In the water of Beishan Wuyi well, the dominant species of $\mathrm{Np}$ is $\mathrm{NpO}_{2}^{+}(93.76 \%)$ and the minor speciations are $\mathrm{NpO}_{2} \mathrm{CO}_{3}^{-}$and $\mathrm{NpO}_{2} \mathrm{OH}(\mathrm{aq})$. The speciation of $\mathrm{Np}$ greatly varies with $\mathrm{pH}$. In strong acid conditions, the dominant is $\mathrm{NpO}_{2}{ }^{+}$and the minor ones are $\mathrm{NpO}_{2}{ }^{2+}$ and $\mathrm{NpO}_{2} \mathrm{SO}_{4}(\mathrm{aq})$; in neutral conditions, the dominant is also $\mathrm{NpO}_{2}{ }^{+}$, but the coexisting ones are $\mathrm{NpO}_{2} \mathrm{CO}_{3}^{-}$and $\mathrm{NpO}_{2} \mathrm{OH}(\mathrm{aq})$; in weak alkaline to strong alkaline conditions, the dominant species change from $\mathrm{NpO}_{2} \mathrm{CO}_{3}{ }^{-}$to $\mathrm{NpO}_{2} \mathrm{OH}(\mathrm{aq})$, and the minor ones are $\mathrm{Np}\left(\mathrm{CO}_{3}\right)_{3}^{4-}$ and $\mathrm{NpO}_{2}\left(\mathrm{CO}_{3}\right)_{2}^{3-}$. Figure 4 gives the detailed evolution.

\subsubsection{Speciation of $\mathrm{Pu}$ as a function of $\mathbf{p H}$}

The calculated species of $\mathrm{Pu}$ were $\mathrm{PuO}_{2}\left(\mathrm{CO}_{3}\right)_{2}{ }^{2-}, \mathrm{PuO}_{2}{ }^{+}$, $\mathrm{PuO}_{2} \mathrm{OH}^{+}, \mathrm{PuO}_{2} \mathrm{~F}_{3}{ }^{-}$and $\mathrm{PuO}_{2} \mathrm{~F}_{2}$ (aq). The speciation of $\mathrm{Pu}$ also varies with $\mathrm{pH}$. The dominant is $\mathrm{PuO}_{2}{ }^{2+}$ and the minor ones are $\mathrm{PuO}_{2} \mathrm{SO}_{4}$ (aq) and $\mathrm{PuO}_{2} \mathrm{~F}^{+}$in strong acid conditions.

Table 3 Speciations of nuclides at $\mathbf{p H} 7.24$

\begin{tabular}{||cc||cc||cc||cc||}
\hline Speciation & Content $(\%)$ & Speciation & Content $(\%)$ & Speciation & Content $(\%)$ & Speciation & Content $(\%)$ \\
\hline $\mathrm{AmCO}_{3}{ }^{+}$ & 84.42 & $\mathrm{PuO}_{2}\left(\mathrm{CO}_{3}\right)_{2}{ }^{2-}$ & 71.88 & $\mathrm{UO}_{2}\left(\mathrm{CO}_{3}\right)_{2}{ }^{2-}$ & 76.75 & $\mathrm{NpO}_{2}{ }^{+}$ & 93.76 \\
$\mathrm{Am}\left(\mathrm{CO}_{3}\right)_{2}{ }^{-}$ & 10.15 & $\mathrm{PuO}_{2}{ }^{+}$ & 23.93 & $\mathrm{UO}_{2}\left(\mathrm{CO}_{3}\right)_{3}{ }^{4-}$ & 12.82 & $\mathrm{NpO}_{2} \mathrm{CO}_{3}^{-}$ & 4.09 \\
$\mathrm{AmOH}^{2+}$ & 2.58 & $\mathrm{PuO}_{2} \mathrm{OH}^{+}$ & 2.13 & $\mathrm{UO}_{2}(\mathrm{OH})_{2}(\mathrm{aq})$ & 7.55 & $\mathrm{NpO}_{2} \mathrm{OH}(\mathrm{aq})$ & 1.84 \\
$\mathrm{AmSO}_{4}{ }^{+}$ & 1.69 & $\mathrm{PuO}_{2} \mathrm{~F}_{3}^{-}$ & 0.802 & $\mathrm{UO}_{2} \mathrm{CO}_{3}(\mathrm{aq})$ & 2.64 & & \\
$\mathrm{Am}^{3+}$ & 0.941 & $\mathrm{PuO}_{2} \mathrm{~F}_{2}(\mathrm{aq})$ & 0.667 & & & $\mathrm{Th}(\mathrm{OH})_{4} \mathrm{aq}$ & 100 \\
\hline
\end{tabular}


Table 4 Saturation indices of some minerals under in-situ condition

\begin{tabular}{|c|c|c|c|c|c|}
\hline Minerals & SI & Minerals & SI & Minerals & SI \\
\hline $\mathrm{PuO}_{2}$ & 3.056 & $\mathrm{UO}_{2} \mathrm{SO}_{4}$ & -22.260 & $\mathrm{UCl}_{4}$ & -82.060 \\
\hline Thorianite & 2.038 & $\mathrm{Pu}\left(\mathrm{OH}_{3}\right.$ & -22.837 & $\mathrm{UCl}_{3}$ & -85.468 \\
\hline $\mathrm{NpO}_{2}$ & 0.801 & UOFOH & -23.121 & $\mathrm{UOBr}_{3}$ & -86.516 \\
\hline $\mathrm{AmOHCO}_{3}$ & -4.535 & $\mathrm{UO}_{2.3333}$ (beta) & -23.313 & $\mathrm{UBrCl}_{3}$ & -89.749 \\
\hline $\mathrm{Pu}(\mathrm{OH})_{4}$ & -5.067 & $\mathrm{UO}_{2} \mathrm{Cl}$ & -23.925 & $\mathrm{UBrCl}_{2}$ & -93.106 \\
\hline $\mathrm{Th}(\mathrm{OH})_{4}$ & -5.755 & $\mathrm{UOF}_{2}$ & -26.053 & $\mathrm{UF}_{6}$ & -93.144 \\
\hline $\mathrm{Np}(\mathrm{OH})_{4}$ & -7.813 & $\mathrm{PuF}_{3}$ & -26.654 & $\mathrm{ThBr}_{4}$ & -94.923 \\
\hline $\mathrm{Am}(\mathrm{OH})_{3}$ & -8.088 & $\mathrm{UO}_{2} \mathrm{SO}_{3}$ & -34.078 & $\mathrm{UO}_{2}\left(\mathrm{NO}_{3}\right)_{2}$ & -95.388 \\
\hline $\mathrm{UO}_{2} \mathrm{CO}_{3}$ & -8.788 & $\mathrm{U}(\mathrm{OH})_{2} \mathrm{SO}_{4}$ & -34.313 & $\mathrm{U}_{2} \mathrm{~F}_{9}$ & -98.153 \\
\hline $\mathrm{NpO}_{2} \mathrm{OH}(\mathrm{am})$ & -9.074 & $\mathrm{UF}_{4}$ & -39.303 & $\mathrm{UBr}_{2} \mathrm{Cl}_{2}$ & -98.580 \\
\hline $\mathrm{Am}(\mathrm{OH})_{3}(\mathrm{am})$ & -9.588 & $\mathrm{PuF}_{4}$ & -39.705 & $\mathrm{UCl}_{5}$ & -101.941 \\
\hline $\mathrm{UO}_{3}($ gamma $)$ & -10.512 & $\mathrm{U}\left(\mathrm{SO}_{4}\right)_{2}$ & -43.353 & $\mathrm{UBr}_{2} \mathrm{Cl}$ & -102.520 \\
\hline $\mathrm{Th}\left(\mathrm{SO}_{4}\right)_{2}$ & -10.776 & $\mathrm{UOCl}_{2}$ & -45.446 & $\mathrm{U}_{3} \mathrm{O}_{5} \mathrm{~F}_{8}$ & -102.885 \\
\hline $\mathrm{UO}_{3}$ (beta) & -11.115 & $\mathrm{U}\left(\mathrm{CO}_{3}\right)_{2}$ & -47.637 & $\mathrm{U}_{2} \mathrm{O}_{2} \mathrm{Cl}_{5}$ & -103.799 \\
\hline $\mathrm{UO}_{3}$ (alpha) & -11.444 & $\mathrm{UClF}_{3}$ & -48.886 & $\mathrm{UBr}_{3} \mathrm{Cl}$ & -107.619 \\
\hline $\mathrm{UO}_{2.25}$ & -12.326 & $\mathrm{Pu}_{2} \mathrm{O}_{3}$ (beta) & -48.907 & $\mathrm{UBr}_{3}$ & -111.104 \\
\hline $\mathrm{ThF}_{4}: 2.5 \mathrm{H}_{2} \mathrm{O}$ & -12.849 & $\mathrm{UO}_{2} \mathrm{Br}_{2}$ & -51.687 & $\mathrm{UBr}_{4}$ & -115.931 \\
\hline $\mathrm{UO}_{2} \mathrm{FOH}$ & -13.115 & $\mathrm{UOF}_{4}$ & -55.985 & $\mathrm{UCl}_{6}$ & -120.674 \\
\hline $\mathrm{ThF}_{4}$ & -14.711 & $\mathrm{UOCl}_{3}$ & -57.170 & $\mathrm{UBr}_{5}$ & -136.954 \\
\hline Uraninite & -15.060 & $\mathrm{UF}_{3}$ & -59.364 & $\mathrm{Th}\left(\mathrm{NO}_{3}\right)_{4}: 5 \mathrm{H}_{2} \mathrm{O}$ & -159.130 \\
\hline $\mathrm{PuO}_{2} \mathrm{OH}(\mathrm{am})$ & -15.263 & $\mathrm{ThC}_{14}$ & -60.135 & $\mathrm{U}_{4} \mathrm{~F}_{17}$ & -175.896 \\
\hline $\mathrm{PuO}_{2}(\mathrm{OH})_{2}$ & -16.589 & $\mathrm{UOBr}_{2}$ & -60.241 & $\mathrm{ThS}_{2}$ & -183.636 \\
\hline $\mathrm{NpO}_{2}(\mathrm{OH})_{2}$ & -18.634 & $\mathrm{UCl}_{2} \mathrm{~F}_{2}$ & -60.785 & $\mathrm{U}_{2} \mathrm{C}_{3}$ & -289.920 \\
\hline $\mathrm{UO}_{2.6667}$ & -18.666 & $\mathrm{UOCl}$ & -62.756 & $\mathrm{Th}_{2} \mathrm{~S}_{3}$ & -335.694 \\
\hline Uranophane & -18.709 & $\mathrm{U}_{5} \mathrm{O}_{12} \mathrm{Cl}$ & -63.234 & $\mathrm{U}_{2} \mathrm{~S}_{3}$ & -356.296 \\
\hline $\mathrm{Np}_{2} \mathrm{O}_{5}$ & -19.176 & $\mathrm{UCl}_{3} \mathrm{~F}$ & -72.509 & $\mathrm{U}_{3} \mathrm{~S}_{5}$ & -387.750 \\
\hline $\mathrm{UO}_{2} \mathrm{~F}_{2}$ & -19.878 & $\mathrm{U}_{2} \mathrm{O}_{3} \mathrm{~F}_{6}$ & -76.014 & $\mathrm{Th}_{7} \mathrm{~S}_{12}$ & -1203.551 \\
\hline $\mathrm{UO}_{2}(\mathrm{am})$ & -20.007 & $\mathrm{U}\left(\mathrm{SO}_{3}\right)_{2}$ & -77.655 & & \\
\hline
\end{tabular}

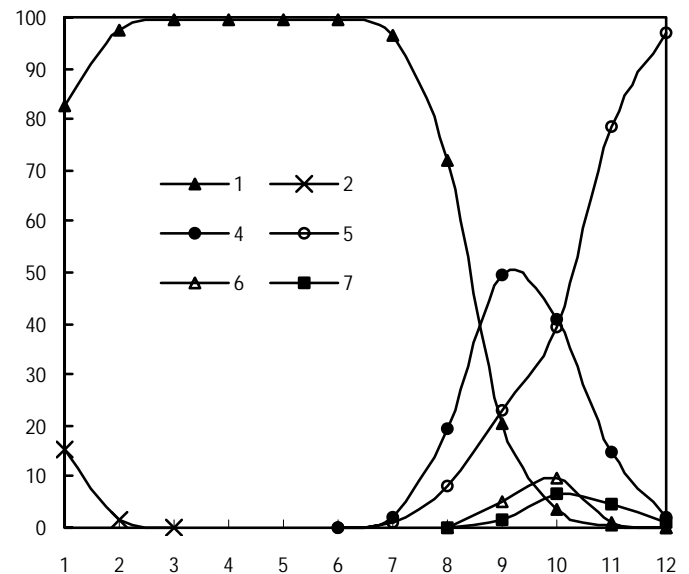

\footnotetext{
1: $\mathrm{NpO}_{2}{ }^{+}{ }^{\mathrm{pH}}$

3: $\mathrm{NpO}_{2} \mathrm{SO}_{4}(\mathrm{aq})$;

4: $\mathrm{NpO}_{2} \mathrm{CO}_{3}^{-}$; 5: $\mathrm{NpO}_{2} \mathrm{OH}(\mathrm{aq})$;

6: $\mathrm{NpO}_{2}\left(\mathrm{CO}_{3}\right)_{3}{ }^{4-} ; \quad 7: \mathrm{NpO}_{2}\left(\mathrm{CO}_{3}\right)_{2}{ }^{3-}$;
}

Fig.4 Speciation of $\mathrm{Np}$ as a function of $\mathrm{pH}$

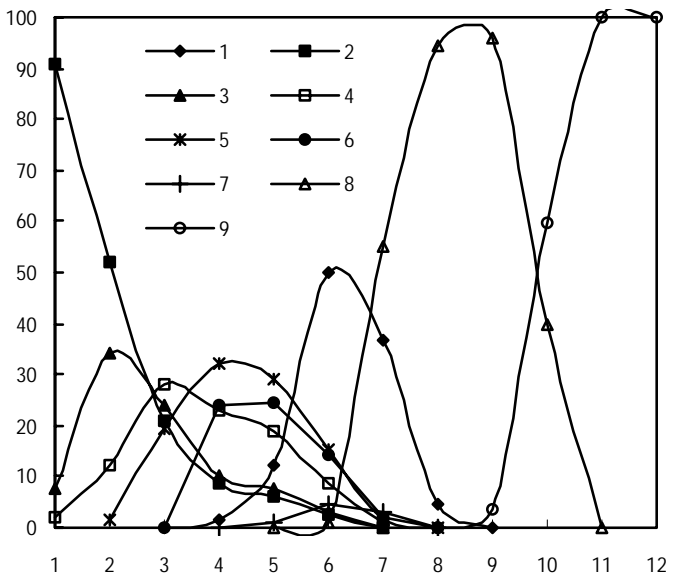

\begin{tabular}{lll} 
& \multicolumn{1}{c}{$\mathrm{pH}$} \\
& \\
1: $\mathrm{PuO}_{2}{ }^{+} ;$ & 2: $\mathrm{PuO}_{2}{ }^{2+} ;$ & 3: $\mathrm{PuO}_{2} \mathrm{SO}_{4}(\mathrm{aq})$ \\
4: $\mathrm{PuO}_{2} \mathrm{~F}^{+} ;$ & 5: $\mathrm{PuO}_{2} \mathrm{~F}_{2}(\mathrm{aq}) ;$ & 6: $\mathrm{PuO}_{2} \mathrm{~F}_{3}^{-} ;$ \\
7: $\mathrm{PuO}_{2} \mathrm{OH}^{+} ;$ & 8: $\mathrm{PuO}_{2}\left(\mathrm{CO}_{3}\right)_{2}{ }^{2-} ;$ & 9: $\mathrm{Pu}(\mathrm{OH})_{5}$
\end{tabular}

Fig . 5 Speciation of $\mathrm{Pu}$ as a function of $\mathrm{pH}$ 


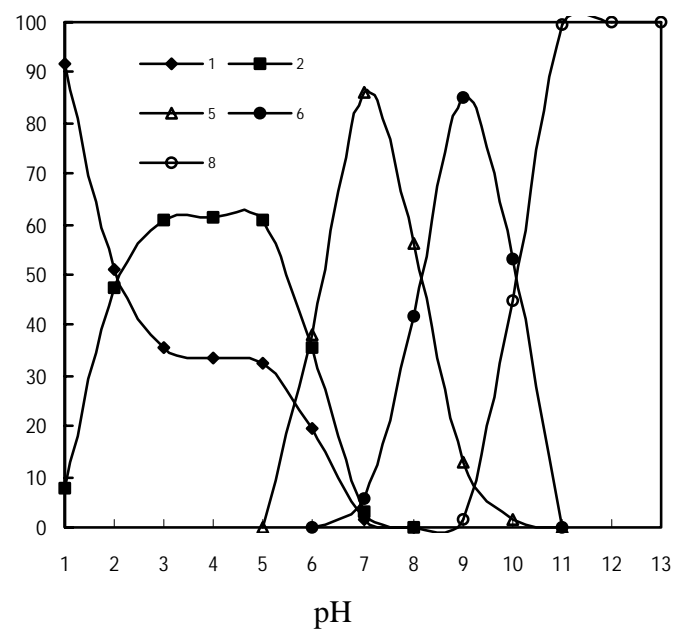
1: $\mathrm{Am}^{3+}$
2: $\mathrm{AmSO}_{4}^{+}$;
3: $\mathrm{Am}\left(\mathrm{SO}_{4}\right)_{2}$
4: $\mathrm{AmF}_{2}^{+}$;
5: $\mathrm{AmCO}_{3}^{+}$;
6: $\mathrm{Am}\left(\mathrm{CO}_{3}\right)_{2}^{-}$;
7: $\mathrm{AmOH}_{2}^{+} ; \quad 8: \mathrm{Am}(\mathrm{OH})_{3}(\mathrm{aq})$

Fig. 6 Speciations of $\mathrm{Am}$ as a function of $\mathrm{pH}$

In neutral conditions, the dominant is $\mathrm{PuO}_{2}\left(\mathrm{CO}_{3}\right)_{2}{ }^{2-}$, but the coexisting ones are $\mathrm{PuO}_{2}{ }^{+}, \mathrm{PuO}_{2} \mathrm{OH}^{+}, \mathrm{PuO}_{2} \mathrm{~F}_{3}{ }^{-}$and $\mathrm{PuO}_{2} \mathrm{~F}_{2}$ (aq).

In weak alkaline to strong alkaline conditions, the dominant species change from $\mathrm{PuO}_{2}\left(\mathrm{CO}_{3}\right)_{2}{ }^{2-}$ to $\mathrm{Pu}(\mathrm{OH})_{5}$. Figuer 5 gives the detailed evolution.

\subsubsection{Speciation of $\mathrm{Am}$ as a function of $\mathrm{pH}$}

In the water of Beishan Wuyi well, the calculated species of Am were $\mathrm{AmCO}_{3}{ }^{+}, \mathrm{Am}\left(\mathrm{CO}_{3}\right)_{2}{ }^{-}, \mathrm{AmOH}^{2+}, \mathrm{AmSO}_{4}{ }^{+}$and $\mathrm{Am}^{3+}$. In strong acid conditions, the dominant species is $\mathrm{Am}^{3+}$; in weak acid conditions, the dominant one is $\mathrm{AmSO}_{4}{ }^{+}$; in neutral conditions, the dominant one is $\mathrm{AmCO}_{3}{ }^{+}$; in weak alkaline the dominant one is $\mathrm{Am}\left(\mathrm{CO}_{3}\right)_{2}{ }^{-}$; in strong alkaline the dominant one is $\mathrm{Am}(\mathrm{OH})_{3}(\mathrm{aq})$. Figure 6 gives the detailed evolution.

\subsubsection{Speciation of $\mathrm{U}$ as a function of $\mathrm{pH}$}

In the groundwater, the calculated $\mathrm{U}$ species were $\mathrm{UO}_{2}\left(\mathrm{CO}_{3}\right)_{2}{ }^{2-}, \mathrm{UO}_{2}\left(\mathrm{CO}_{3}\right)_{3}{ }^{4-}, \mathrm{UO}_{2}(\mathrm{OH})_{2}(\mathrm{aq})$ and $\mathrm{UO}_{2} \mathrm{CO}_{3}(\mathrm{aq})$. When the $\mathrm{pH}$ changes from low to high , the dominant species of $\mathrm{U}$ is changed from $\mathrm{UO}_{2}{ }^{2+}$ to $\mathrm{UO}_{2} \mathrm{CO}_{3}(\mathrm{aq}), \mathrm{UO}_{2}\left(\mathrm{CO}_{3}\right)_{2}{ }^{2-}$, $\mathrm{UO}_{2}\left(\mathrm{CO}_{3}\right)_{3}{ }^{4-}$, and finally to $\mathrm{UO}_{2}(\mathrm{OH})_{3}{ }^{-}$. The detailed evolution is given in Fig .7.

\subsubsection{Speciation of $\mathrm{Th}$ as a function of $\mathrm{pH}$}

The speciation of Th differs from the above nuclides. $\mathrm{Th}(\mathrm{OH})_{4}(\mathrm{aq})$ is the dominant species of $\mathrm{Th}$ when the $\mathrm{pH}$ is higher than 6. However in acid conditions, it changes with the $\mathrm{pH}$, and the dominant species changes from $\mathrm{ThSO}_{4}{ }^{2+}$ to $\mathrm{Th}$ $\left(\mathrm{SO}_{4}\right)_{2}(\mathrm{aq})$, then $\mathrm{ThF}_{2}{ }^{2+}$, and finally to $\mathrm{Th}(\mathrm{OH})_{4}(\mathrm{aq})$ with increasing $\mathrm{pH}$.

The speciation pattern of $\mathrm{Th}$ is different from other Actinides for the lack of thermodynamic data on Th carbonates as shown in Table1. Figure 8 shows the detailed evolution.

\section{CONCLUSIONS}

The following conclusions can be derived from the calculation

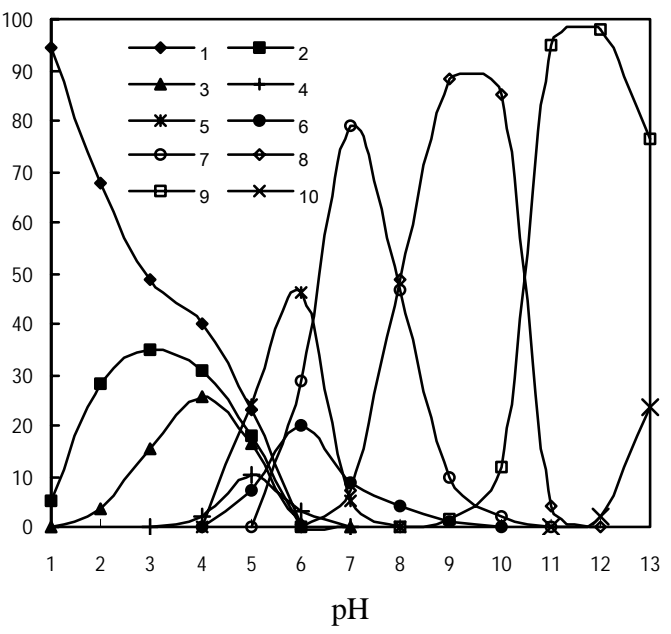

1: $\mathrm{UO}_{2}{ }^{2+}$;

2: $\mathrm{UO}_{2} \mathrm{SO}_{4}(\mathrm{aq}) ; \quad 3: \mathrm{UO}_{2} \mathrm{~F}$ 4: $\mathrm{UO}_{2} \mathrm{OH}$

5: $\mathrm{UO}_{2}\left(\mathrm{CO}_{3}\right) \mathrm{aq} ; \quad 6: \mathrm{UO}_{2}(\mathrm{OH})_{2} \mathrm{aq} ; \quad 7: \mathrm{UO}_{2}\left(\mathrm{CO}_{3}\right)_{2}{ }^{2-}$; $8: \mathrm{UO}_{2}\left(\mathrm{CO}_{3}\right)_{3}{ }^{4-} ; \quad 9: \mathrm{UO}_{2}(\mathrm{OH})_{3}{ }^{-} ; \quad 10: \mathrm{UO}_{2}(\mathrm{OH})_{4}{ }^{2-}$

\section{Fig . 7 Speciations of $\mathrm{U}$ as a function of $\mathbf{p H}$}

The speciations of $\mathrm{Np}, \mathrm{Pu}, \mathrm{Am}, \mathrm{U}$ and $\mathrm{Th}$ are affected not only by the chemical composition of the water but also by the $\mathrm{pH}$ value even with the same chemical composition.

Each of the five nuclides has its own speciation pattern, but the speciation of $\mathrm{Pu}, \mathrm{Am}, \mathrm{U}$ and Th is very complicated in acid conditions.

The speciation of nuclides is simpler in alkaline conditions, and the dominant species are hydroxyl complexes and carbonate complexes. It is thus important to study the adsorption and migration behavior of these species in geological materials.

The SI values of $\mathrm{PuO}_{2}$, Thorianite and $\mathrm{NpO}_{2}$ are higher than zero in Beishan Wuyi well. Those minerals would be precipitated and should not dissolve over $10^{-12} \mathrm{~mol} / \mathrm{L}$ at that condition. Other minerals such as $\mathrm{AmOHCO}_{3}$ are hardly formed and the solubility will be higher than $10^{-12} \mathrm{~mol} / \mathrm{L}$.

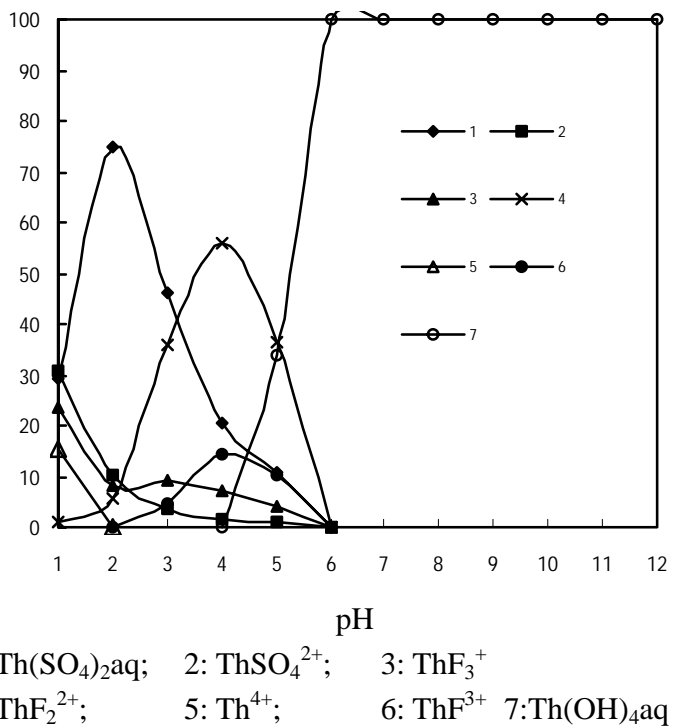

Fig.8 Speciations of $\mathrm{Th}$ as a function of $\mathrm{pH}$ 


\section{REFERENCES}

[1] Xu, G. Q., et al.: Deep geological disposal of high level radioactive waste in China. In: Geological Problem in Radioactive Waste Isolation: Second Worldwide Review (Witherspoon, P.A. ed), pp.51-61(1996).

[2] Wolery, T. J.: EQ3/6, A software package for geochemical modeling of aqueous systems: package overview and installation guide (version 7.0), (1992).

[3] Wolery, T. J.: EQ3NR, A computer program for geochemical aqueous speciation-solubility calculations: theoretical manual, user's guide, and related documentation (version 7.0), (1992).

[4] Yang, T. X., Guo, Y.H.: The Ground Water Chemical Characteristics of Beishan Area-The China's Potential High-Level Radioactive Waste Repository. Acta Geoscientia Sinica. 20, 679-685(1999). 